\title{
Study on Wave Field Characteristics and Imaging of Collapse Column in Three-Dimensional Detection with Love Channel Wave Reflected outside the Working Face
}

\author{
Huachao Sun 1*, Huide Zhang², Jinyun Wang3, Xianzhuang Lv, Xin Ding1, Shiyu Xing4, Heng Zhang4 \\ ${ }^{1}$ China University of Mining and Technology, School of Resources and Geophysics, Xuzhou, China \\ ${ }^{2}$ Shang Dong Energy Linyi Group Luxi Coalmine, Jining, China \\ ${ }^{3}$ Kaijia Group Yitang Changyuan Coalmine, Jiexiu, China \\ ${ }^{4}$ China University of Mining and Technology, School of Mechanics and Civil Engineering, Xuzhou, China \\ Email: *shc0627@126.com
}

How to cite this paper: Sun, H.C., Zhang, H.D., Wang, J.Y., Lv, X.Z., Ding, X., Xing, S.Y. and Zhang, H. (2020) Study on Wave Field Characteristics and Imaging of Collapse Column in Three-Dimensional Detection with Love Channel Wave Reflected outside the Working Face. Open Journal of Geology, 10, 1027-1039.

https://doi.org/10.4236/ojg.2020.1011048

Received: October 1, 2020

Accepted: November 6, 2020

Published: November 9, 2020

Copyright $\odot 2020$ by author(s) and Scientific Research Publishing Inc. This work is licensed under the Creative Commons Attribution International License (CC BY 4.0).

http://creativecommons.org/licenses/by/4.0/

\begin{abstract}
The safety accidents caused by collapse column water diversion occur frequently, which has great hidden danger to the safety production of coal mine. Limited by the space of underground, the detection of collapse column on the outside of working face has been a difficult problem. Based on this, numerical simulation and imaging research were carried out in this paper. The results indicate that when a seismic source near the roadway is excited, a part of seismic wave propagates along the roadway direction, namely direct P-wave, direct $S$-wave and direct Love channel wave. When the body waves and Love channel wave propagating to the outside of working face meet the interface of collapse column, the reflected Love channel wave and reflected body waves are generated. Reflection body waves and direct waves are mixed in time domain, which is difficult to identify in seismic records, while reflected Love channel wave whose amplitude is relatively strong. The reflected Love channel wave which has a large interval from other wave trains in the time domain is easily recognizable in seismic record, which makes it suitable for advanced detection of collapse column. The signal-to-noise ratio of $\mathrm{X}$ component is higher than that of $\mathrm{Y}$ component and $\mathrm{Z}$ component. According to the seismic records, polarization filtering was carried out to enhance the effective wave, which removed the interference waves, and the signal was migrated to get the position parameters of collapse column interface, which was basically consistent with the model position.
\end{abstract}




\section{Keywords}

Reflected Love Channel Wave, 3D Numerical Simulation, Collapse Column, Wave Field Characteristics, Migration Imaging

\section{Introduction}

Collapse column is a typical geological structure in coal mining in North and South China. Its formation is mainly related to tectonic fissure zone, fracture, groundwater activity, and karst [1]. Due to the frequent occurrence of safety accidents caused by collapse column conducting water, there exist huge hidden dangers to the safety production of coal mine [2]. Therefore, the detection of collapse column has been a hot topic. With the increase of coal mining depth, the accuracy of three-dimensional seismic exploration on the ground has been limited [3]. Seismic detection in coal mining is not affected by ground conditions and has the advantages of being close to the geological structure, less attenuation of the energy of seismic waves and high-frequency components and interference of seismic wave propagation path [4], and is then applied in structure detection of coal mine [5]. At the same time, there is a special geological body of coal seam in the coal mine. Therefore, channel wave detection is the preferred method of seismic detection [6].

Transmission channel wave method is the most typical method of collapse column detection [7]. When the source is excited in the coal seam, the presence of the collapse column will destroy the continuity of the coal seam, and the non-development of the channel wave is one of the signs of the existence of the collapse column [8]. In 2015, Wang Ji used transmission channel wave detection for in-face collapse column detection. Based on the channel wave energy attenuation coefficient for imaging research, the boundary position of the collapse column was circled and was relatively consistent with the actual results [9]. Li Guangyi et al. used channel wave seismic transmission method to detect the collapse column in field and circled the range of the collapse column, which effectively guided the actual production [10]. In 2019, Ma Xin et al. studied the collapse column of the coal mining work face detected by the transmission channel wave through numerical simulation [11].

Mason et al. conducted an experimental study on the detection of faults in working face using reflected channel wave in the Pye Hill coal mine. Buchanan proposed dynamic trace gathering (DTG) [12]. Buchanan then proposed dynamic gather stacking and adaptive delayed summation method [13]. Elsen and Schott conducted reflected channel wave experiments at several coal mines in Germany, including Patberg and Stein, and used polarization filtering and envelope superposition for imaging [14]. $\mathrm{Hu}$ et al. introduced reverse-time pre-stack elastic migration technology into the study of reflected channel wave, and formed the image of reflected wave by means of model data [15]. However, 
relatively less research has been done on the detection of collapse column outside the working face.

Based on the above problems, this paper uses numerical simulation to study the detection of collapse column by using reflected Love channel wave outside the working face. The purpose of this study is to reveal the three-dimensional wave field characteristics of collapse column detection, and use feature waves for inverse imaging research, which provides theoretical guidance for the detection of collapse column in underground coal seam.

\section{Principle of Channel Wave Detection and Imaging}

\subsection{Principle of Sidewall Reflected Channel Wave}

The direct P-wave and S-wave are first received in seismic records, subsequently, the direct channel wave also propagates to the receivers and is received. This three direct wave groups are the most obvious. When there is structural development in the working face (there is a wave impedance interface), which generated reflected channel wave [16], and the detection diagram is shown in Figure 1. Therefore, the development and distribution of the internal structure of the working face are detected by reflected channel wave.

\subsection{Principle of Polarization Migration Imaging}

In a certain time window, the covariance matrix is formed according to the amplitude value set of spatial data points, and the eigenvalues and corresponding eigenvectors of the covariance matrix are solved. The eigenvector corresponding to the maximum eigenvalue must be in the maximum polarization direction. According to the particle polarization and the relationship between the principal polarization direction and the propagation path direction, the filtering parameters

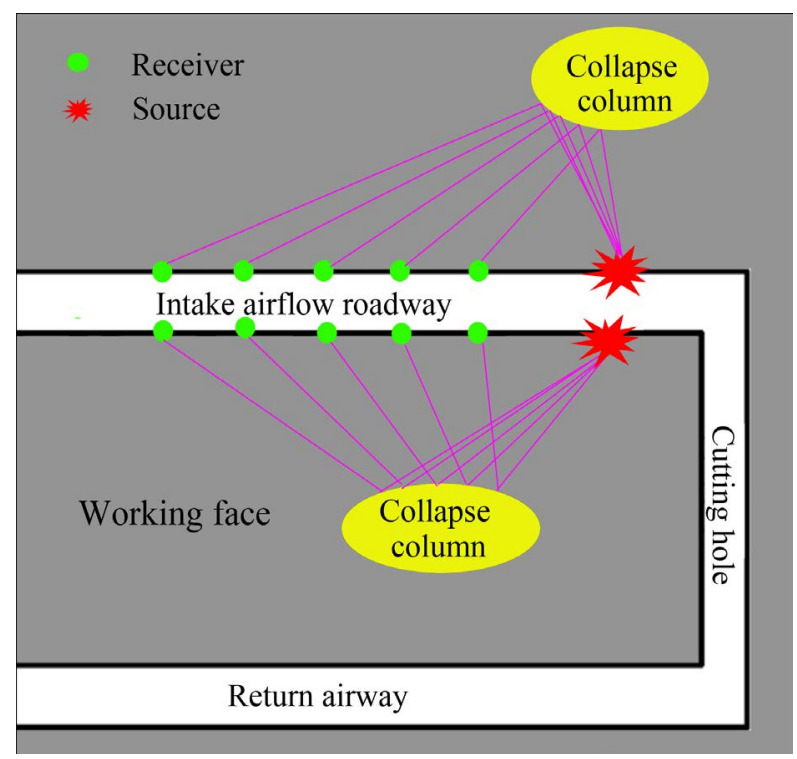

Figure 1. Schematic diagram of reflected channel wave detection. 
are set to separate the effective wave from the interference wave [17].

1) The constitution of covariance matrix

For three-component data acquisition, assume that there are $\mathrm{m}$ sampling points in the time window $\left(t_{1}, t_{2}\right)$, and the three-components on each receiving point correspond to $X_{i}, Y_{i}, Z_{i}$ (representing the amplitude value of the sampling point, respectively), and their average values in the time window $\left(t_{1}, t_{2}\right)$ are as follows:

$$
N_{x}=\frac{1}{M} \sum_{i=M_{1}}^{M_{2}} X_{i}, \quad N_{y}=\frac{1}{M} \sum_{i=M_{1}}^{M_{2}} Y_{i}, \quad N_{z}=\frac{1}{M} \sum_{i=M_{1}}^{M_{2}} Z_{i}
$$

where $\left(M_{2}-M_{1}\right) \cdot \Delta t=t_{2}-t_{1}, \quad M=M_{2}-M_{1}+1, \Delta t$ is the sampling interval, and the above formula can be sorted into covariance matrix:

$$
\frac{1}{M}\left[\begin{array}{l}
\sum_{i=M_{1}}^{M_{2}}\left(X_{i}-N_{x}\right)\left(X_{i}-N_{x}\right), \sum_{i=M_{1}}^{M_{2}}\left(X_{i}-N_{x}\right)\left(Y_{i}-N_{y}\right), \sum_{i=M_{1}}^{M_{2}}\left(X_{i}-N_{x}\right)\left(Z_{i}-N_{z}\right), \\
\sum_{i=M_{1}}^{M_{2}}\left(Y_{i}-N_{y}\right)\left(X_{i}-N_{x}\right), \sum_{i=M_{1}}^{M_{2}}\left(Y_{i}-N_{y}\right)\left(Y_{i}-N_{y}\right), \sum_{i=M_{1}}^{M_{2}}\left(Y_{i}-N_{y}\right)\left(Z_{i}-N_{z}\right), \\
\sum_{i=M_{1}}^{M_{2}}\left(Z_{i}-N_{z}\right)\left(X_{i}-N_{x}\right), \sum_{i=M_{1}}^{M_{2}}\left(Z_{i}-N_{z}\right)\left(Y_{i}-N_{y}\right), \sum_{i=M_{1}}^{M_{2}}\left(Z_{i}-N_{z}\right)\left(Z_{i}-N_{z}\right),
\end{array}\right]
$$

According to the covariance matrix, three eigenvalues, $\lambda_{1}, \lambda_{2}$ and $\lambda_{3}$ can be obtained. Through these three eigenvalues, the axis of the ellipsoid composed of the particle vibration track can be determined. The principal eigenvector $V_{1}$ corresponding to the maximum eigenvalue $\lambda_{1}$ is the long axis direction of the ellipsoid, which is called the principal polarization direction.

2) Parameter setting and selection of polarization filter

According to the eigenvalues $\lambda_{1}, \lambda_{2}$ and $\lambda_{3}$ obtained from the covariance matrix and the angle $\theta$ between the principal polarization direction and the propagation path direction, the polarization coefficient factor $\tau$ and the principal polarization direction factor $\varphi$ are defined.

Polarization coefficient factor $\tau$.

$$
\tau=\frac{\left(1-\frac{\lambda_{2}}{\lambda_{1}}\right)^{2}+\left(1-\frac{\lambda_{3}}{\lambda_{1}}\right)^{2}+\left(\frac{\lambda_{2}}{\lambda_{1}}-\frac{\lambda_{3}}{\lambda_{1}}\right)^{2}}{2\left(1+\frac{\lambda_{2}}{\lambda_{1}}+\frac{\lambda_{3}}{\lambda_{1}}\right)^{2}}
$$

principal polarization direction factor $\varphi$ :

$$
\varphi= \begin{cases}\cos \theta, & \mathrm{P} \text {-wave } \\ \sin \theta, & \text { S-wave }\end{cases}
$$

The filtering function is set as follows:

$$
F(t)=\tau^{p}(t) \varphi^{q}(t)
$$

where, the indexes $P$ and $Q$ are the weighted coefficients.

The original seismic record is the arrival time information about the spatial position and reflection phase of the excitation receiver. Generally, the reflection 
path information in these original records cannot directly represent the actual location of the underground geological body, so the subsequent migration and homing processing is needed [18].

If the S-coordinate of the source's point is $\left(x_{s}, y_{s}, z_{s}\right)$, the R-coordinate of the receiver's point is $\left(x_{R}, Y_{R}, z_{R}\right)$, and the G-coordinate of the reflection point is $\left(x_{G}\right.$ $y_{G} z_{G}$ ), then the ellipsoid equation formed by the source-receiver and the travel path of the reflected signal is as follows:

$$
\frac{x_{G}^{2}}{L_{G}^{2}-4 z_{S}^{2}}+\frac{y_{G}^{2}}{L_{G}^{2}-4 z_{S}^{2}}+\frac{z_{G}^{2}}{L_{G}^{2}}=\frac{1}{4}
$$

According to the principle of pre-stack diffraction migration, each source-receiver can form an ellipsoid according to the travel length of seismic ray. Due to the multiple coverage technology in the actual detection, there are many pairs of source and check relations. At this time, each pair of source and check relations can construct the ellipsoid equation, and all the ellipsoid equations are combined to obtain the common section, which is the position where the reflection signal is generated.

\section{Numerical Modeling and Analysis}

\subsection{Numerical Modeling}

According to the result of the three-dimensional seismic exploration showed that there may be a large collapse column about $90 \mathrm{~m}$ in diameter at the distance of $60 \mathrm{~m}$ from the outside of a working face in actual engineering project. However, the surface terrain is complex and the fluctuations are large, which caused large restriction and low reliability of the three-dimensional seismic detection. In order to ensure the safety of the working face, detection work must be carried out in the coal mine. As the collapse column is located outside the boundary of the working face, there is only one roadway around it. Limited by the construction conditions, the working face transmission detection cannot be done, therefore, only the reflected channel wave detection is a suitable choice.

Taking into account the field conditions, a three-dimensional simulation was conducted based on high-order staggered-grid finite difference method [19], and a three-dimensional model of the collapse column containing the roadway was designed, as is shown in Figure 2, and the elastic parameters of the collapse column model are shown in Table 1 . The physical parameters of this model meet the excitation conditions of Love channel wave [20]. A three-dimensional spatial coordinate system was established, $\mathrm{X}$ direction is vertical to the survey line, that is, the direction of coal seam side; $\mathrm{Y}$ direction is parallel to the survey line, that is, the direction pointing to the fault; $\mathrm{Z}$ direction is vertical downward, that is, the direction pointing to the floor. The size of the model in the $\mathrm{X}, \mathrm{Y}$ and $\mathrm{Z}$ directions is $200 \mathrm{~m}, 400 \mathrm{~m}, 200 \mathrm{~m}$, respectively. The width of air roadway is $4 \mathrm{~m}$, and the height is $5 \mathrm{~m}$. The central position was located at the coordinate point $0 \mathrm{~m}, 2.5 \mathrm{~m}$ and $97.5 \mathrm{~m}$ in $\mathrm{X}, \mathrm{Y}$, and $\mathrm{Z}$ direction. The length is $400 \mathrm{~m}$ and the coal thickness is $2.5 \mathrm{~m}$. The collapse column model boundary is located in 


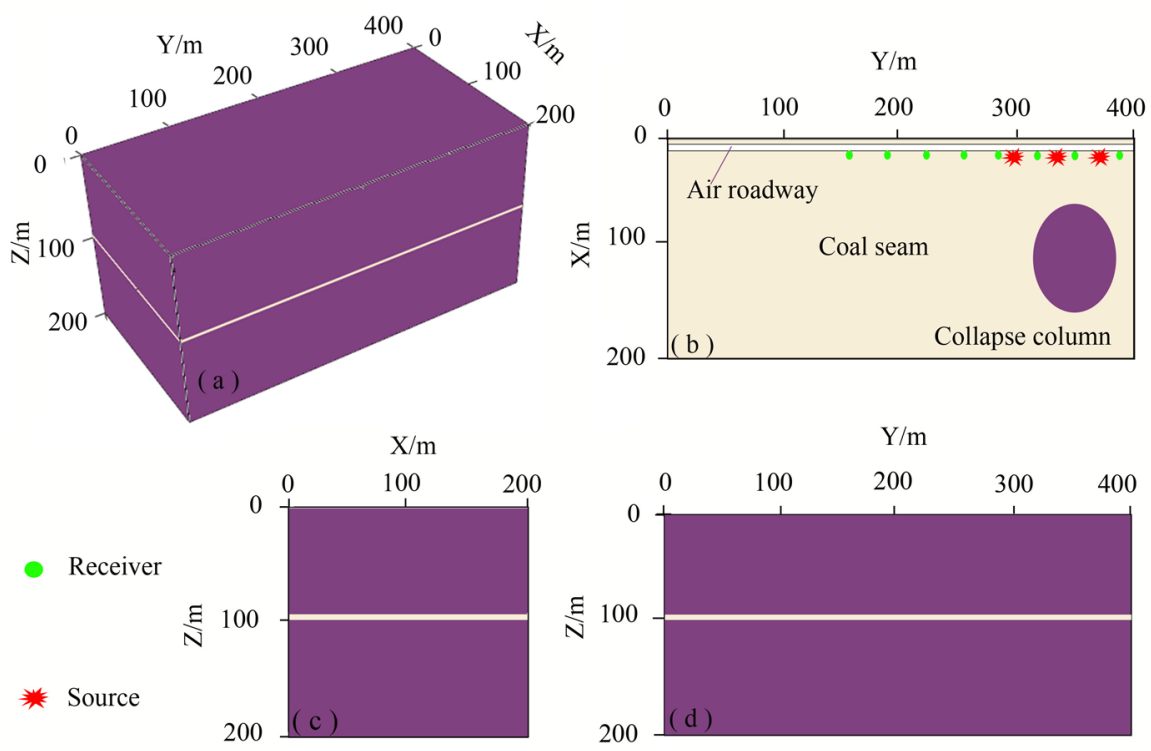

Figure 2. Numerical model. (a) 3D-model diagram; (b) top view; (c) left view; (d) front view.

Table 1. Model parameters.

\begin{tabular}{cccc}
\hline Medium & $V_{p} /\left(\mathrm{m} \cdot \mathrm{s}^{-1}\right)$ & $V_{s} /\left(\mathrm{m} \cdot \mathrm{s}^{-1}\right)$ & $\rho /\left(\mathrm{kg} \cdot \mathrm{m}^{-3}\right)$ \\
\hline Surrounding rock & 4100 & 2369 & 2365 \\
Coal seam & 1800 & 1050 & 1300 \\
Air roadway & 340 & 0 & 1.29 \\
Collapse column & 2700 & 1560 & 2200 \\
\hline
\end{tabular}

the X direction $302 \mathrm{~m}$ to $390 \mathrm{~m}, \mathrm{Y}$ direction $67 \mathrm{~m}$ to $187 \mathrm{~m}, \mathrm{Z}$ direction $85 \mathrm{~m}$ to $140 \mathrm{~m}$. The size of the model grid is $\Delta \mathrm{x}=\Delta \mathrm{y}=\Delta \mathrm{z}=0.2 \mathrm{~m}$.

The observation system was arranged with 25 three-component receivers and the offset was $10 \mathrm{~m}$. The receivers are arranged in a straight line in the direction of the collapse column. The position of NO1 receiver was located at the coordinate point $5 \mathrm{~m}, 5 \mathrm{~m}$ and $98 \mathrm{~m}$ in $\mathrm{X}, \mathrm{Y}$, and $\mathrm{Z}$ direction. There are 3 sources totally, and the position of source $S 1$ was located at the coordinate point $390 \mathrm{~m}, 5 \mathrm{~m}$ and $98 \mathrm{~m}$ in $\mathrm{X}, \mathrm{Y}$, and $\mathrm{Z}$ direction. The position of source S2 was located at the coordinate point $350 \mathrm{~m}, 5 \mathrm{~m}$ and $98 \mathrm{~m}$ in $\mathrm{X}, \mathrm{Y}$, and $\mathrm{Z}$ direction. The position of source S3 was located at the coordinate point $300 \mathrm{~m}, 5 \mathrm{~m}$ and $98 \mathrm{~m}$ in $\mathrm{X}, \mathrm{Y}$, and $\mathrm{Z}$ direction. The numerical simulation is carried out using $\mathrm{X}$-direction concentration source with a source frequency of $120 \mathrm{~Hz}$ and a sampling frequency of 10 $\mathrm{kHz}$. The model boundary uses PML absorption boundary [21], and the free interface of roadway is processed by HA method to reduce the interference of artificial boundary [22].

\subsection{Seismic Record}

Figures 3(a)-(c) respectively show the $\mathrm{X}$ component seismic records of $\mathrm{S} 1$ source, S2 source and S3 source. As is shown, Event 1, Event 2, Event 3 stand for 

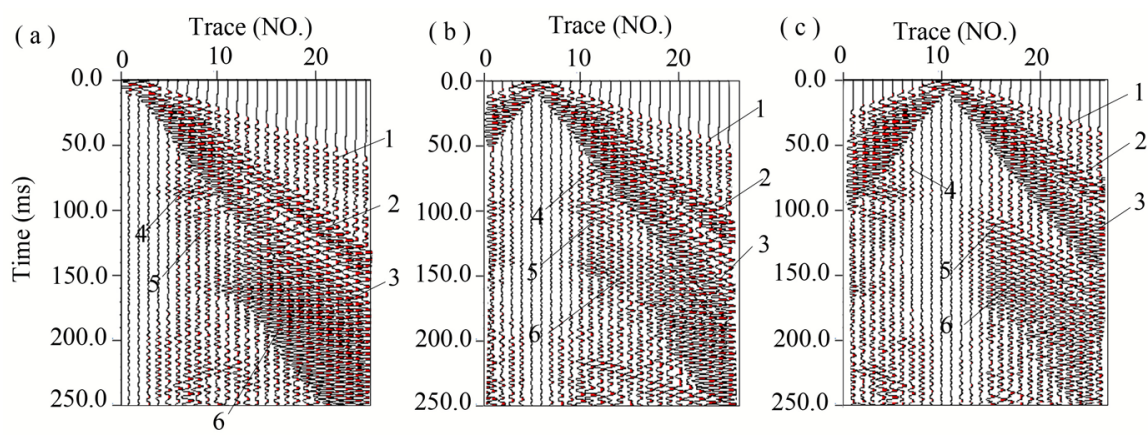

Figure 3. Seismic record of X component, (a) S1 source seismic record, (b) S2 source seismic record, and (c) S3 source seismic record.

direct P-wave, direct S-wave, and direct channel wave. When the seismic wave propagates in the impedance interface of collapse column, it reflects back. Hence, Event 4, Event 5, and Event 6 stand for the reflected P-wave, reflected $\mathrm{S}$-wave, and reflected channel wave. The seismic records of $\mathrm{Y}$ and $\mathrm{Z}$ components are shown in Figure 4.

Through comparison, it shows that the signal-to-noise ratio of the $\mathrm{X}$ component is higher than that of the $\mathrm{Z}$ component and $\mathrm{Z}$ component. Love channel wave is formed by $\mathrm{SH}$ wave interference [23], and $\mathrm{SH}$ wave is relatively developed in $\mathrm{X}$ component, so the energy of Love channel wave in $\mathrm{X}$ component is relatively stronger. At the same time, the reflected wave of S2 source is distinguished obviously, and the hyperbola is obvious. The signal-to-noise ratio is relatively higher.

\subsection{Analysis of Three Dimensional Snapshots of Wave Field}

According to the seismic records, the signal-to-noise ratio of $\mathrm{X}$ component is higher than that of $\mathrm{Y}$ component and $\mathrm{Z}$ component. Therefore, the wave field snapshot of $\mathrm{X}$ component of $\mathrm{S} 2$ source is selected to analyze the characteristics of seismic waves. Figure 5 shows the wave field snapshots of $\mathrm{X}$ component at 30 $\mathrm{ms}, 60 \mathrm{~ms}$ and $70 \mathrm{~ms}$. At $30 \mathrm{~ms}$, wave front 1 , wave front 2 and wave front 3 are direct $\mathrm{P}$-wave, direct S-wave and direct Love channel wave. when the seismic waves propagate to $60 \mathrm{~ms}$, seismic waves meet the wave impedance interface of collapse column, and part of seismic waves propagate into the collapse column, wave front 4 is transmission $\mathrm{P}$-wave, wave front 5 is transmission S-wave, and part of seismic wave generates reflected waves, wave front 6 is reflected P-wave propagating in the direction of roadway, and wave front 7 is reflected S-wave. At $70 \mathrm{~ms}$, when the Love channel wave propagates to the impedance interface of the collapse column, a part of the Love channel wave propagates into the collapse column. Because there is no coal seam in the collapse column, the Love channel wave is converted into SH wave [24], and continues to propagate along the collapse column. As shown in wave front 8 , wave front 9 is the reflected Love channel wave.

Figure 6 shows the wave field snapshots of X component at $90 \mathrm{~ms}, 100 \mathrm{~ms}$ 


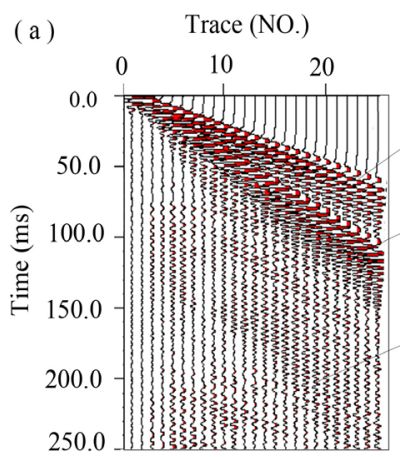

( c)
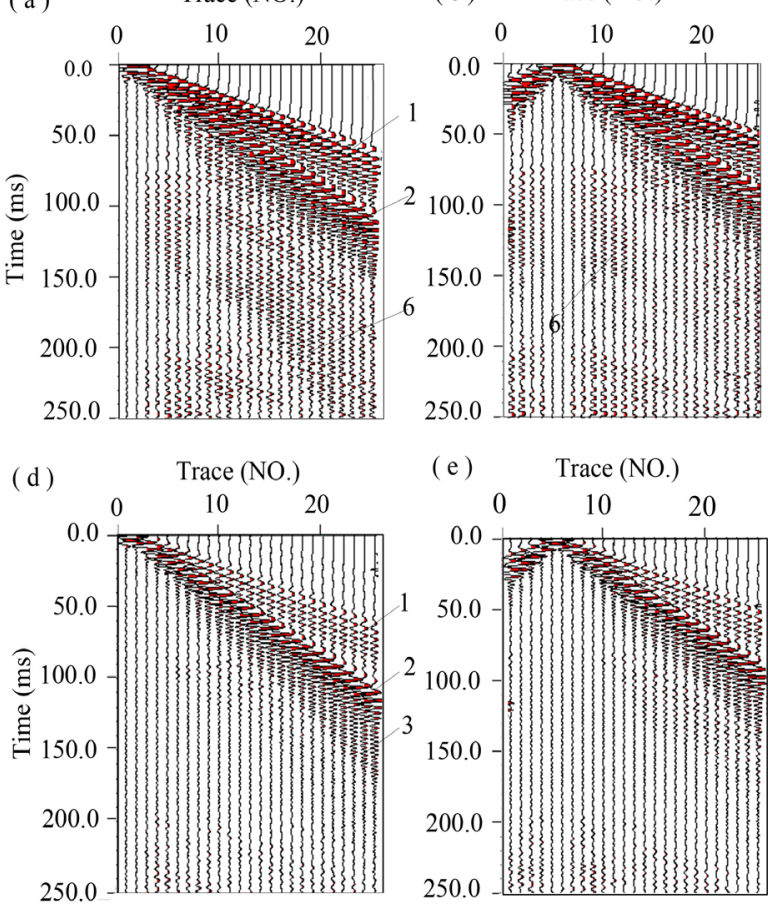

(e) Trace (NO.)

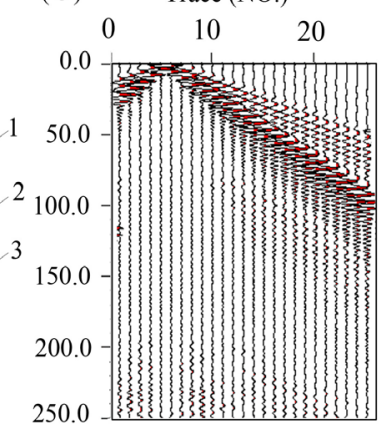

(d) Trace (NO.)

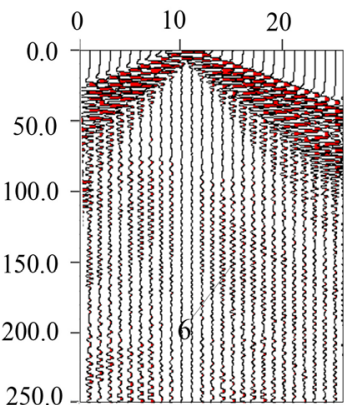

(f) Trace (NO.)

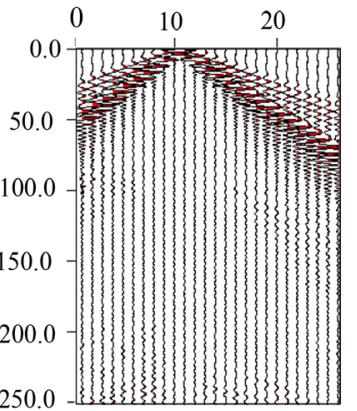

Figure 4. Seismic records, (a) shows the Y-component seismic record of S1 source, (b) shows the Y-component seismic record of S2 source, (c) shows the Y-component seismic record of S3 source; (d) shows the Z-component seismic record of S1 source, (e) shows the z-component seismic record of S2 source, (f) shows the z-component seismic record of S3 source.
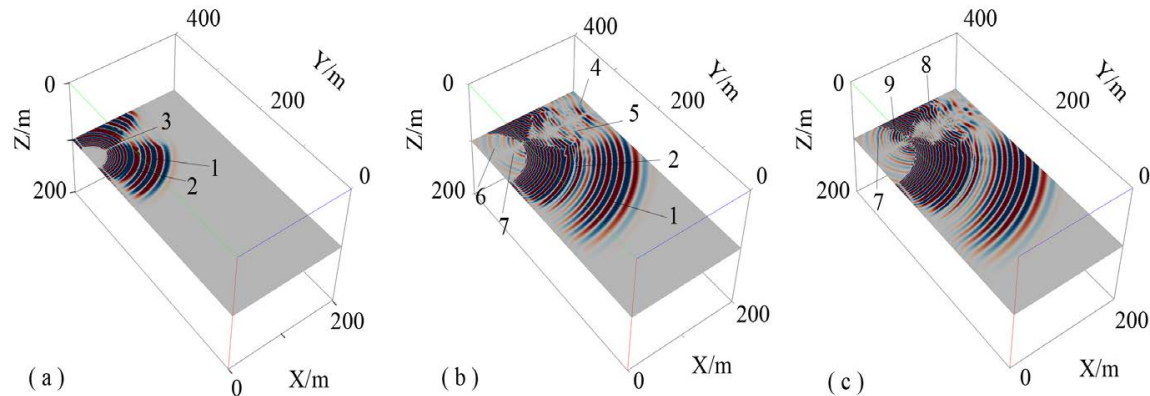

Figure 5. 3D wave field snapshot, (a) $30 \mathrm{~ms}$ wave field snapshot, (b) $60 \mathrm{~ms}$ wave field snapshot, (c) $70 \mathrm{~ms}$ wave field snapshot.
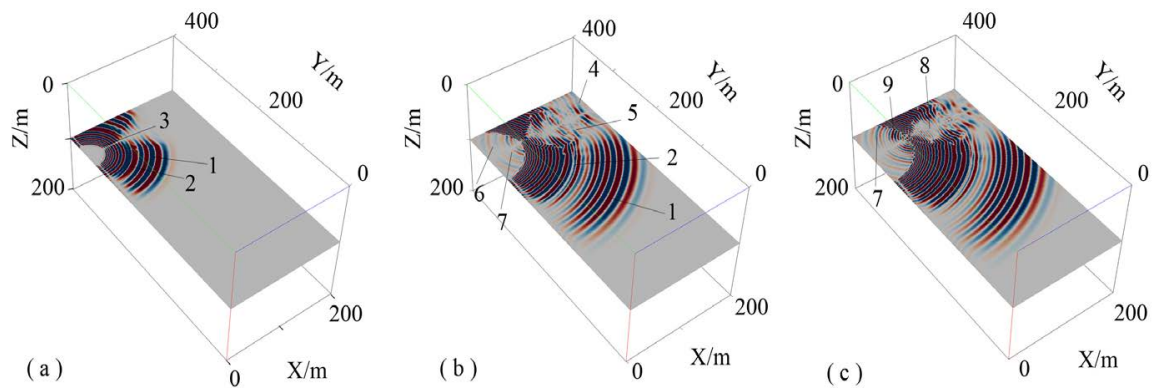

Figure 6. 3D wave field snapshot, (a) $90 \mathrm{~ms}$ time wave field snapshot, (b) $100 \mathrm{~ms}$ time wave field snapshot, (c) $120 \mathrm{~ms}$ wave field snapshot. 
and $120 \mathrm{~ms}$. When the seismic waves propagate to $90 \mathrm{~ms}$, the reflected body waves and the direct waves are mixed, reflected S-wave and reflected Love channel wave, as shown in wavefront 8 and wavefront 9 . The wave field snapshots at $100 \mathrm{~ms}$ and $120 \mathrm{~ms}$ are similar to that at $90 \mathrm{~ms}$, but with the increase of propagation distance, reflected S-wave is mixed with direct Love channel wave.

To sum up, the source is excited near the roadway, the direct P-wave, S-wave and slot wave propagate along the roadway and are received by the receivers. When P-wave, S-wave and Love channel wave propagate to the collapse column interface, which generate reflected P-wave, reflected S-wave, and Love channel wave. But some reflected P-wave and reflected S-wave are easily mixed with direct channel wave, so it is difficult to distinguish them from the seismic record. Therefore, the reflection trough is reflected in the time domain. The wave is the characteristic wave of collapse column.

\section{Research on Inversion Imaging}

The Y-component signal with the highest signal-to-noise ratio is used for migration imaging. Firstly, the original signal is preprocessed. After polarization filtering, the interference waves are removed and the effective wave is enhanced. The processing result is shown in Figure 7. The reflected Love channel wave is shown as event 1 , and the reflected Love channel wave signal is enhanced.

The velocity of Love channel wave is $1.1 \mathrm{~km} / \mathrm{s}$, and the result of migration imaging is shown in Figure 8, which is basically consistent with the forward model. Due to the limitation of the space of the roadway, only part of the collapse column boundary can be detected. The experimental results provide theoretical guidance for the next field test.

\section{Discussions}

\subsection{Comparison between Seismic Detection Method of Channel Wave and Body Waves}

In seismic detection of working face by using the reflected body waves and the

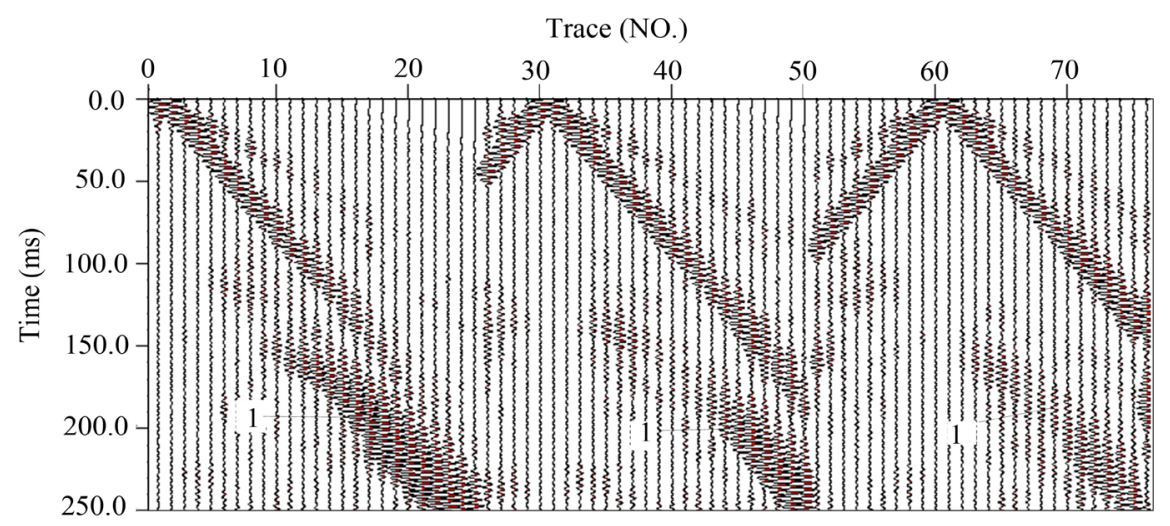

Figure 7. Three component seismic record after polarization filtering by S2 source, (a) X component seismic record, (b) Y component seismic record, and (c) Z component seismic record. 


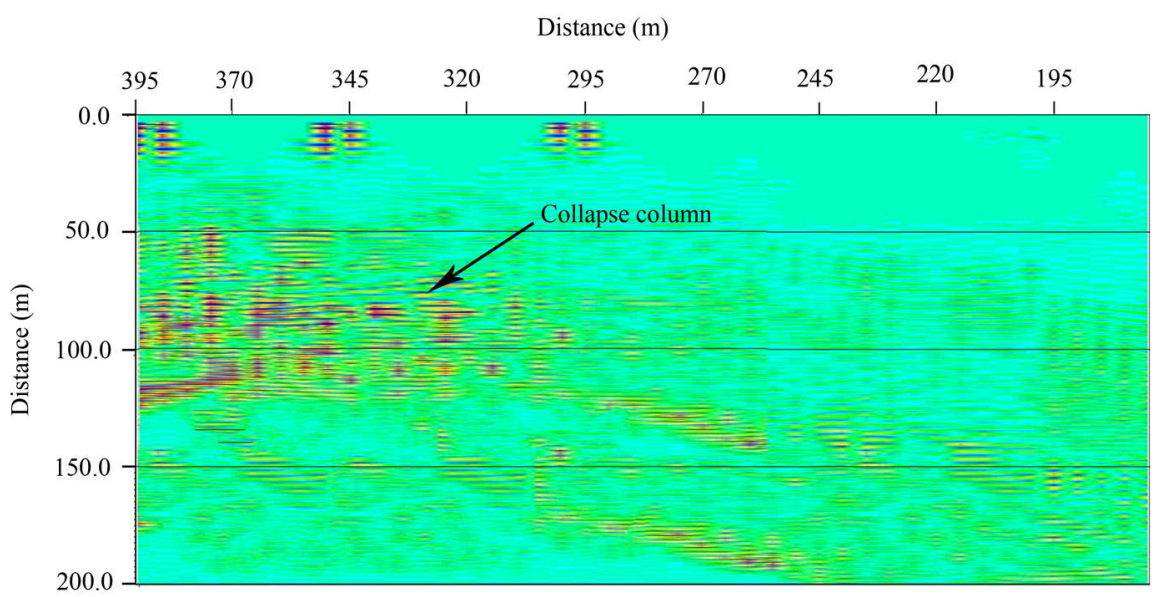

Figure 8. Migration imaging.

reflected channel wave, when the source is excited in the coal seam, most of the energy will be confined in the coal seam [25], so the body waves energy is relatively weak, and the channel wave energy is relatively strong. At the same time, in the conventional reflection detection, the reflected body waves are easy to mix with the channel wave, which reduces the signal-to-noise ratio of seismic records. Relatively speaking, the energy of channel wave is strong and the wave train is wide, which is distinguished from other wave trains in time domain. Meanwhile, the high frequency signal of the channel wave is rich. Compared with the body waves, the imaging resolution of the channel wave is higher.

\subsection{Comparison with Detection Method of Rayleigh Channel Wave and Love Channel Wave}

Channel waves can be divided into two types: Love channel waves and Rayleigh channel waves. The development condition of Love channel wave is relatively simple, but the formation condition of a Rayleigh channel wave is quite strict [26]. When the parameters of surrounding rock and coal seam do not meet the excitation conditions of Rayleigh channel wave, effective signals cannot be collected. The generation condition of Love channel wave (perpendicular to coal wall) is simple and more universal. From the perspective of actual construction, it is easier to operate the exciting and receiving in the left and right sides of the coal roadway than in the roof and floor.

\section{Conclusions}

The following conclusions are obtained through numerical simulation:

1) The three-dimensional numerical simulation of the collapse column model on the outside of the working face shows that the reflected Love channel wave's amplitude is relatively strong. The Love wave which has a large interval from other wave trains in the time domain is easily recognizable in seismic record. The signal-to-noise ratio of $\mathrm{X}$ component is higher than that of $\mathrm{Y}$ component and $\mathrm{Z}$ component. 
2) When a seismic source near the roadway is excited, a part of seismic wave propagates along the roadway direction, namely direct $\mathrm{P}$-wave, direct S-wave and direct Love channel wave. When the body waves and Love channel wave propagating to the outside of working face meet the interface of collapse column, the reflected Love channel wave and reflected body waves are generated. Reflection body waves and direct waves are mixed in time domain, which is difficult to identify in seismic records, but the reflected Love channel wave is easy to identify on seismic records due to the large interval other wave trains in time domain. The reflected Love channel wave is a characteristic wave existing at the interface of collapse column.

3) According to the seismic records, polarization filtering is carried out to enhance the effective wave, remove the interference waves, which removes the interference wave, and the signal is migrated to get the position parameters of collapse column interface, which is basically consistent with the model position. The research results provide theoretical guidance for the next step of field test.

\section{Acknowledgements}

This paper is supported by the National Natural Science Foundation of China (Nos. 41604082, 51734009, 51904270, 41904118), the Independent Innovation Project for Double First-level Construction (China University of Mining and Technology) (No. 2018ZZCX04) and the National Key R\&D Program of China (No. 2018YFC0807802).

\section{Conflicts of Interest}

The authors declare no conflicts of interest regarding the publication of this paper.

\section{References}

[1] Wu, Q., Cui, F.P., Zhao, S.Q., Liu, S.Q., Zeng, Y.F. and Gu, Y.W. (2013) Type Classification and Main Characteristics of Mine Water Disasters. Journal of China Coal Society, 4, 561-565. https://doi.org/10.13225/j.cnki.jccs.2013.04.015

[2] Dong, S.N. (2010) Some Key Scientific Problems on Water Hazards Frequently Happened in China's Coal Mines. Journal of China Coal Society, 35, 66-71. https://doi.org/10.13225/j.cnki.jccs.2010.01.019

[3] Chang, Z.J., Wei, W., Fu, L.Y., Qin, N., Su, J. and Sun, W.J. (2020) Focal Beam Resolution Analysis of Wide-Band, Wide-Azimuth and High-Density Land 3D Seismic Acquisition Geometries. Chinese Journal of Geophysics, 63, 3868-3885. https://doi.org/10.6038/cjg2020N0114

[4] Yuan, L. and Zhang, S.P. (2019) Development Status and Prospect of Geological Guarantee Technology for Precise Coal Mining. Journal of China Coal Society, 44, 2277-2284. https://doi.org/10.13225/j.cnki.jccs.KJ19.0571

[5] Liu, S.D., Liu, J. and Yue, J.H. (2014) Development Status and Key Problems of Chinese Mining Geophysical Technology. Journal of China Coal Society, 39, 19-25. https://doi.org/10.13225/j.cnki.jccs.2013.0587

[6] Cheng, J.L., Li, F. and Peng, S.P. (2014) Research Progress and Development Direc- 
tion on Advanced Detection in Mine Roadway Working Face Using Geophysical Methods. Journal of China Coal Society, 39, 1742-1750. https://doi.org/10.13225/j.cnki.jccs.2014.9007

[7] Ji, G.Z., Cheng, J.Y., Hu, J.W., Wang, J., Li, G. and Wang, B.L. (2014) In-Seam Wave Imaging Using Attenuation Coefficient: Method and Application. Journal of China Coal Society, 39, 471-475. https://doi.org/10.13225/j.cnki.jccs.2013.1672

[8] Hu, G.Z., Teng, J.W., Pi, J.L., Wang, W. and Qiao, Y.H. (2013) In-Seam Seismic Exploration Techniques-A Geophysical Method Predicting Coal-Mine Disaster. In: Rabinovich, A.B., Ed., Progress in Geophysics, Springer, Berlin, 439-451.

[9] Wang, J., Li, J.Z., Wu, H. Wang, B.L., Hu, J.W. and Ji, G.Z. (2015) Tomography of Transmission In-Seam Wave Attenuation Coefficient and Detection of Collapse Columns. Coal Science and Technology, 43, 108-111.

https://doi.org/10.13199/j.cnki.cst.2015.01.026

[10] Li, G.Y., Jiao, Y., Wei, J.S., Tan, J., Dou, W.W. and Li, Z.Y. (2018) Application of Channel Wave Seismic Transmission Method in Detection of Collapse Columns. Shanxi Coal, 38, 35-38. https://doi.org/10.3969/j.issn.1672-5050.2018.06.009

[11] Ma, X., Yang, S.T., Li, X.F., Guo, X.D., Bai, J.L., Wen, Z.J. and Zhang, L. (2019) Simulation Study on Detection of Collapse Column in Coal Face Based on Transmitted Slot Wave. Safety in Coal Mines, 50, 32-36. https://doi.org/10.13347/j.cnki.mkaq.2019.04.008

[12] Mason, I.M., Buchanan, D.J. and Booer, A.K. (1980) Fault Location by Underground Seismic Survey. Communications Radar \& Signal Processing IEE Proceedings $F$, 127, 322-336. https://doi.org/10.1049/ip-f-1.1980.0048

[13] Buchanan, D.J., Davis, R., Jackson, P.J. and Taylor, P.M. (1981) Fault Location by Channel Wave Seismology in United Kingdom Coal Seams. Geophysics, 46, 994-1002. https://doi.org/10.1190/1.1441248

[14] Dresen, L. and Ruter, H. (1994) Seismic Coal Exploration, Part B: In-Seam Seismic. Pergamon Press, New York, 388-394.

[15] Hu, Y.R. and Mcmechan, G.A. (2007) Imaging Mining Hazards within Coalbeds Using Prestack Wave Equation Migration of In-Seam Seismic Survey Data: A Feasibility Study with Synthetic Data. Journal of Applied Geophysics, 63, 24-34. https://doi.org/10.1016/j.jappgeo.2007.03.002

[16] Ji, G.Z. (2017) Diffraction Migration Imaging of Reflected In-Seam Waves and Its Application. Coal Geology \& Exploration, 45, 121-124. https://doi.org/10.3969/j.issn.1001-1986.2017.01.024

[17] Liu, S.D., Zhang, J., Li, C.Y., Wang, B., Jin, B. and Liu, J.S. (2019) Method and Test of Mine Seismic Multi Wave and Multi-Component. Journal of China Coal Society, 44, 271-277.

[18] Ye, Y.F., Sun, J.G., Liu, Z.B., Zhang, Y.M., Sun, W.B. and Tong, Z.F. (2012) Conversion of Seismic Data Offset by Series Kirchhoff Migration and Demigration. Chinese Journal of Geophysics, 69, 3783-3790. https://doi.org/10.6038/cjg2018M0070

[19] Ji, G.Z., Chen, J.Y., Zhu, P.M. and Li H. (2012) 3-D Numerical Simulation and Dispersion Analysis of In-Seam Wave in Underground Coal Mine. Chinese Journal of Geophysics, 55, 645-654.

[20] Chen, J.Y., Ji, G.Z. and Zhu, P.M. (2012) Love Channel-Waves Dispersion Characteristic Analysis of Typical Coal Models. Journal of China Coal Society, 37, 67-72.

[21] Mezafajardo, K.C. and Papageorgiou, A.S. (2008) A Nonconvolutional, Split-Field, Perfectly Matched Layer for Wave Propagation in Isotropic and Anisotropic Elastic 
Media: Stability Analysis. Bulletin of the Seismological Society of America, 98, 1811-1836. https://doi.org/10.1785/0120070223

[22] Xu, Y., Xia, J. and Miller, R.D. (2007) Numerical Investigation of Implementation of Air-Earth Boundary by Acoustic-Elastic Boundary Approach. Geophysics, 72, 147-153. https://doi.org/10.1190/1.2753831

[23] Wang, B.L., Zhang, H.L. and Zhang, Q.Q. (2016) Frequency Domain Synthesis Algorithm of Love Type Channel Wave Transmission Record. Coal Geology of China, 28, 73-77. https://doi.org/10.3969/j.issn.1674-1803.2016.06.15

[24] Huang, L.Y., Wang, S.C. and Song, X.J. (2020) Three-Component Channel Wave Characteristics and Fine Imaging of Erosion Zone. In: Al-Dossary, O.M., Ed., Journal of King Saud University Science, Elsevier, Amsterdam, 1197-1205.

https://doi.org/10.1016/j.jksus.2019.10.012

[25] Yang, S.T. and Chen, J.L. (2012) The Method of Small Structure Prediction Ahead with Rayleigh Channel Wave in Coal Roadway and Seismic Wave Field Numerical Simulation. Chinese Journal of Geophysics, 55, 655-662.

[26] Wang, B., Sun, H.C., Huang, L.Y., Liu, S.D., Jin, B., Zhang, H., Zhang, Z.D., Ding, X., Qiu, W.Y. and Wang, S.C. (2020) Wave Field Characteristics of Small Faults around the Loose Circle of Rock Surrounding a Coal Roadway. Journal of Environmental and Engineering Geophysics, 25, 245-254. 